\title{
Interdépendances territoriales et résiliaires en France de l'Est
}

Interdependence of territories and networks in eastern France

Wechselbeziehungen zwischen Territorien und Netzen in Ostfrankreich

\section{Henri Nonn}

\section{(2) OpenEdition}

\section{Journals}

Édition électronique

URL : http://journals.openedition.org/rge/636

ISSN : 2108-6478

Éditeur

Association des géographes de l'Est

Édition imprimée

Date de publication : 1 juin 2004

Pagination : 107-115

ISSN : 0035-3213

Référence électronique

Henri Nonn, « Interdépendances territoriales et résiliaires en France de l'Est », Revue Géographique de I'Est [En ligne], vol. 44 / 3-4 | 2004, mis en ligne le 04 juin 2009, consulté le 07 septembre 2020. URL : http://journals.openedition.org/rge/636

Ce document a été généré automatiquement le 7 septembre 2020

Tous droits réservés 


\title{
Interdépendances territoriales et résiliaires en France de l'Est
}

\author{
Interdependence of territories and networks in eastern France \\ Wechselbeziehungen zwischen Territorien und Netzen in Ostfrankreich
}

Henri Nonn

\section{NOTE DE L'ÉDITEUR}

Article reçu le 10 juillet 2004, accepté le 5 septembre 2004

Des processus subis, tendanciels ou volontaristes orientent les recompositions et les dynamiques territoriales actuelles en France de l'Est, comme ailleurs. Les ajustements qui les expriment se lisent à différents niveaux, selon des logiques "territoriales» (ancrages aux lieux, infléchissements de trames structurantes, évolutions dans les besoins de proximité, recherche de meilleure cohérence...), et selon des logiques de " réseaux » par lesquels les territoires "respirent", s'articulent à des ensembles voisins, englobants, ou plus extérieurs. Les transformations s'opèrent à la fois en fonction de conditions et d'enjeux d'ordre "général » (ex: intégration européenne, internationalisation de l'économie, réorganisation administrative nationale...), en fonction de «volontés régionales » de développement interne équilibré, et en fonction d'attentes locales plus spécifiées et souvent distinctes à courte distance, comme inégalement exprimées ou mobilisatrices d'énergies endogènes. A ces titres, le Nord-Est français se prête remarquablement à une analyse s'attachant aux interdépendances d'échelles, de démarches, de logiques et d'enjeux influençant les organisations territoriales existantes ou que l'on tente de susciter. 


\section{Une France de l'Est « interrégionale »?}

2 Le concept d'interrégion (en contiguiité) est né d'une réflexion et d'un effort d'orientation de la politique nationale d'aménagement du territoire dans les années 1990 sous l'impulsion de la DATAR ${ }^{1}$. Poussaient à reconsidérer les démarches antérieures: l'amplification de la mondialisation économique, la dynamique communautaire (U.E.), la compétition lancée par de grandes entités "régionales » fortes de leurs poids humains, économiques et résiliaires supérieurs à la plupart des Régions françaises de province (ici, Lorraine: 2,3 millions d'habitants, Alsace et Bourgogne: 1,6; Franche-Comté et Champagne-Ardenne: moins de 1,35 en 1990)². Rapprocher, coordonner des énergies et des ressources des Régions, rationaliser les actions de l'État, mieux peser dans les régions d'Europe, tels étaient les objectifs.

3 De fait, institutionnellement, les Régions en France étaient financièrement peu dotées, n'avaient alors que des capacités d'initiative limitées, et partagées : l'État restait maître de la définition des axes majeurs de programmation, de la régulation et du contrôle sur les compétences à elles allouées, et maintenait l'absence de tutelle d'une collectivité locale sur une autre. Les Régions disposaient surtout d'une fonction de coordination et d'impulsion, conditionnée par les modalités de transfert de compétences et de moyens (souvent en financements " croisés»), laquelle impliquait, pour la validation de leur rôle de " chef de file ", une solide capacité d'entraînement et d'obtention de consensus.

Économiquement, si tant est qu'y existaient des "tissus régionaux», ceux-ci étaient bousculés ou en ajustements; et individuellement chaque Région pesait assez peu : en pourcentage de valeur ajoutée brute nationale (1996), la Lorraine comptait pour 3,4, l'Alsace 3, la Bourgogne 2,4, Champagne-Ardenne 2,1, la Franche-Comté 1,7 (source : La France dans ses régions, SEDES, 3e éd. 2000). En réalité, des liaisons et organisations d'entreprise (banques, firmes, délégations interrégionales, réseaux de sous-traitance...) dépassaient déjà alors leurs cadres pendant que, aux plans décisionnels et d'impulsion, des grandes métropoles économiques s'imposaient. Les frontières devenues très perméables jouaient en outre sensiblement à deux niveaux : accès aux marchés des pays voisins par des implantations d'établissements ou acquisitions, et exploitation de «différentiels » locaux avantageux. A ces titres, la France de l'Est participa tôt de ces mutations. Enfin, les restructurations de pans entiers d'industries faisaient largement dépendre d'investissements nationaux - et communautaires - les destinées de territoires en crise (sidérurgie, charbon, potasse, textile).D'autre part, l'État, face à des difficultés budgétaires accrues, est amené à réduire les saupoudrages d'équipements publics, au moins dans ses domaines régaliens : enseignement supérieur et recherche, transports, etc. et à les rendre plus compétitifs dans une concurrence internationale durcie.

5 Partant des distributions des grands ensembles de peuplement, de l'identification d'une dizaine de «métropoles de taille européenne » ayant d'assez vastes réseaux, la DATAR a proposé de considérer en prospective 7 ensembles interrégionaux dénommés "grands chantiers » où l'on estimait que des composantes structurelles, des enjeux partiellement partagés, pouvaient susciter des coordinations pertinentes et efficace (gabarits, ressources), favoriser des rapprochements entre État, élus et société civile.

6 Cependant, l'interrégionalité proposée a rencontré plusieurs obstacles:dans l'appareil d'État central, maintes fois; dans les Régions montant en puissance et en capacité de négociation (contractualisations multipliées) élaborant leur propre prospective: ici 
« Lorraine 2003 », « Alsace 2005 » et « Franche-Comté 2005 », et surtout préoccupées de réduire leurs écarts internes de ressources et de dynamiques territoriales, soucieuses aussi de voir pris en compte leurs propres choix au regard des enjeux nationaux et européens (cf: Débat national pour l'aménagement du territoire, Document d'étape, 1994) : ici en misant sur d'autres interrégionalités que celles de la proximité, là en s'investissant également dans la démarche $\mathrm{d}^{\text {' }}$ eurorégions ${ }^{3}$; et les mobilisations des milieux économiques et sociaux se sont révélées inégales. Aussi, une relance récente (1998 -2002), dans le cadre de 6 MIIAT (missions interministérielles et interrégionales d'aménagement du territoire) s'adresse essentiellement aux services de l'État et vise des objectifs plus sélectifs, intégrant les Schémas de services publics nés de la LOADDT de 1999 et les préconisations du Schéma de Développement de l'Espace communautaire (SDEC) établi en 1997-99, tout en escomptant un entraînement plus large (élus, société civile $)^{4}$.

7 Le « Grand Est »est l'un de ces " grands chantiers » des années 1990-96, et le cadre d'une MIIAT récente, englobant l'Alsace, la Lorraine, la Franche-Comté, la Bourgogne et la Champagne-Ardenne : soit, en 1990 et en 1999, 13,8 \% de la population nationale. On y souligne quelques traits partagés à même d'étayer des coordinations, des solidarités et des synergies :

1. une même appartenance à une aire géographique "intermédiaire " entre foyers majeurs de développement francilien comme rhône-alpin et "Dorsale européenne " constituée du chapelet de grandes métropoles et de régions économiques fortes allant de la Mer du Nord et l'Italie du Nord par le Rhin et la Suisse du Nord - cependant les degrés de participation à leurs dynamiques y varient en intensité ou en orientation - ;

2. un long passé de "régions périphériques", économiquement dominées, vouées aux activités et emplois de " production » ou de "gestion", et au cours duquel bien des sièges de décision locaux ont été perdus ou absorbés, les emplois tertiaires moins étoffés.. ;

3. des économies récentes soutenues amplement par des investissements extérieurs de firmes nationales et étrangères, intéressées par l'ouverture des frontières, les marchés internationaux, les savoir-faire professionnels acquis, les capacités de sous-traitance... ;

4. une intégration à la géographie des flux (et des infrastructures) d'échanges marchands ouesteuropéens, aux hinterlands des ports du «Northern Range", à l'utilisation du couloir rhodanien ;

5. le partage d'une "culture de l'effort et de l'adversité» (Datar, 2002, p. 17) forgée au long d'une Histoire maintes fois douloureuse.

8 De «périphérique ", le Grand Est est à même de changer de statut et d'avenir, en devenant " interface active » à la faveur des "portes » qu'elle détient sur une Union européenne consolidée et élargie qui se veut «polycentrique » (SDEC) et d'une participation mieux établie à des systèmes résiliaires étendus et multiples.

9 Toutefois, un tel ensemble n'est ni sans failles ni sans faiblesses. Sa partie occidentale reste sous l'attractivité francilienne, et sa partie méridionale sous celle de Lyon. Ce qui a le plus de liens internes est un "noyau dur $»^{5}$ fait de l'Alsace, de la Lorraine et de la Franche-Comté (liens dans l'histoire économique et réseaux plus récents, échanges universitaires, scientifiques, transferts technologiques) ; là sont les « portes » (Moselle, Rhin supérieur, Porte de Bourgogne), tandis que les deux autres Régions ne lui sont solidaires que pour certains grands dossiers (TGV, logistique des transports, valorisation des patrimoines et tourisme par ex.). Et, même au sein du «noyau dur », demeurent des « contraintes d'architectures »: géographie naturelle ; 
organisations urbaines régionales inégalement complètes et sans affirmation d'une grande métropole intégratrice; des trames d'infrastructures mises en place en fonction d'autres primats (v. infra, § 2) et avec des déficiences de connexions internes. En outre, les relations transfrontalières et « eurorégionales » actuelles sont peu convergentes (v. §3).

10 Les instances nationales comprennent assez mal que la mobilisation sur l'interrégional soit incomplète ou limitée au regard des enjeux, et à l'heure où se dilatent les échelles d'organisation et de relations. Sans doute cela provient-il d'une sous-évaluation qu'elles font des héritages, d'une perception externe insuffisamment attentive aux évolutions territoriales actuelles qui y accentuent les écarts à courtes distances quant aux bases et aux dynamismes de développement dans ses composantes, au point de justifier la priorité donnée par les instances régionales et locales aux questions de cohérence et de recompositions internes. Et les orientations transfrontalières plus volontiers aujourd'hui mobilisatrices relèvent surtout de rapports nécessaires et de réalisme, aidés des opportunités offertes par les programmes Interreg. Essayons de préciser.

\section{Des territoires régionaux très différenciés, y compris dans leurs recompositions actuelles}

11 (On s'en tiendra ici au seul « noyau dur ».)

\section{Le poids des héritages structurels reste " prégnant » et « imprégnant »}

On ne remontera pas aux temps de constitution des entités provinciales, encore qu'en subsistent des éléments de références identitaires, pour ne considérer que la période contemporaine (XIXe et 1re moitié du XXe siècle).

La mise en place des réseaux de transportsa privilégié le niveau national : la capitale, les bassins industriels de Loire et du Creusot, pour les premières voies ferrées et fluviales, en recoupant les axes naturels ou de commerce d'antan. Les grandes articulations ferroviaires internes, les raccordements principaux aux réseaux étrangers voisins comme à Lyon, aux bassins industriels du Nord et de la Sarre, ne sont réalisés qu'entre 1856 et 1870 , ou plus tard'. Plusieurs de ces communications seront presque aussitôt entravées par la frontière de 1871, qui bloquera aussi les relations transvosgiennes. Les dessertes locales n'étoffent les réseaux qu'en fonction des besoins économiques, en laissant d'importantes zones d'ombre; d'autres ont des intérêts surtout militaires. L'Entre-deux-guerres n'apportera que des améliorations techniques - sauf pour le Rhin, voie importante de redistribution marchande.

Les grandes organisations économiques (capitalistiques, de marchés...) s'affirment dans un cadre national - bientôt protectionniste - où le Nord-Est subit les handicaps des distances-temps (et coûts), la concurrence de régions plus spécialisées que lui dans l'agriculture et l'élevage, la petite industrie. En outre, pour l'Alsace-Moselle, c'est l'obligation, de 1871 à 1919, de repositionner ses marchés avec de nouvelles concurrences; ce sont d'importants départs de forces vives, de capitaux et de sièges, une coupure politico- administrative forte. Si cela lui vaut des ouvertures sarro- 
rhénanes, sont perdues des relations antérieures avec les Vosges lorraines textiles et avec le Territoire de Belfort disjoint de l'Alsace; en réaction, un appui sur les bases endogènes s'effectue dans la durée. L'unicité nationale rétablie (sauf pour l'AlsaceMoselle de 1940 à 1944) n'apporte que peu d'inflexions favorables à l'interrégionalité, sinon par la politique rhénane française qui permet l'articulation entre la sidérurgie lorraine et le Port de Strasbourg et amplifie le rôle de redistribution de celui-ci sur une partie des régions de l'Est. Les liens issus des replis industriels proches de la frontière de 1871 sont maintenus. Néanmoins, les «Marches de l'Est» seront exclues des implantations d'industries stratégiques et de haute technologie, et subiront dès les années 30 les effets d'une politique « de glacis » tarissant maints investissements.

Territorialement, se constituent à cette époque des «bassins industriels » spécifiques délimités (fer et acier, charbon, potasse). En Lorraine, la sidérurgie suscite en fait 3 sous-ensembles cloisonnés par la frontière et par les différences d'intérêts et de stratégies des groupes industriels. Et, même après 1919, hors productions de base, les bassins ne connaîtront que peu de diversifications en aval. Avec des activités plus diverses, s'étoffe également le foyer industriel de Belfort - Montbéliard et environs. Bref, autant de territoires "particuliers»: par leurs peuplements, leurs emplois (et statuts), leurs habitats, leurs mentalités et pratiques sociales ou styles de vie, leurs paysages... ${ }^{7}$ A l'opposé, s'étiolent plusieurs " pays » d'industries traditionnelles dont les villes-centres sont en stagnation: ceci sans que l'économie générale et la gestion publique contribuent à créer de plus amples cohérences ou systèmes d'atténuation des écarts.

16 Les organisations urbaines ne jouent pas non plus ce rôle ${ }^{8}$. Les agglomérations majeures construisent chacune leur propre système de développement. Strasbourg et Nancy établissent le leur sur des activités d'industries diverses, sur leur carrefour, leurs fonctions commerciales et bancaires, des universités et grandes écoles de renom, des équipements et services supérieurs attractifs (N.B. : Strasbourg a été aussi capitale du « Reichsland d'Alsace-Lorraine » de 1871 à 1919). Mulhouse voit son «modèle » de pôle industriel amoindri (bases, structures, encadrement, impulsions). Metz aussi subit un repli, généré par l'annexion, sur des fonctions tertiaires concurrencées et sur une fonction militaire contraignante. Besançon n'a d'assises qu'un commandement sur un département, qu'une université modeste, et ses industries, principalement horlogères, alors que l'essor de « la Porte d'Alsace » (B. Dézert) et l'autonomie organisationnelle des pays du Jura du Sud réduisent ses champs d'influence. Quant aux "villes moyennes ", elles se partagent: "pôles industriels» (sous-tertiarisés, tels Thionville, Longwy, Montbéliard...), « centres de services » locaux (Altkirch, Haguenau, Vesoul...), et « villes mixtes ", les unes à industries stagnantes (Neufchâteau, Commercy...), les autres relancées par de nouvelles usines (Pont-à-Mousson, Lunéville, Thann..) - à côté de rôles administratifs de sous-préfectures auxquels s'adossent fréquemment des composantes de centralité (commerce, banque, presse...). Le tout dans des cadres départementaux solides, alors que les services et équipements supérieurs des grandes villes sont encore souvent destinés à certaines catégories de populations seulement. 


\section{Les évolutions plus contemporaines élargissent, modifient, recomposent les horizons}

17 Les maillages en grand des systèmes de transports, à compter des années 50, sont améliorés. Les autoroutes prennent corps après 1970. En liaisons Ouest-Est, l'A4 (1976) relie à Paris le Bas-Rhin et la Moselle du Nord qui, par elle, sont aussi connectés à la Sarre et au Benelux (années 80); mais son tracé est très septentrional; des routes nationales assurent toujours partiellement les relations de Nancy à l'Alsace ou aux villes champenoises. L'A36 Bâle-Mulhouse-Beaune ou Dijon (par Besançon) date de 1981; mais la RN 19 est encore seule à relier la Porte d'Alsace à Langres ou Troyes vers Paris et vers le couloir de la Loire. En trames Nord-Sud, la toute récente A 39 (1998) ne fait qu'effleurer l'Ouest du Jura. L'A 32-31 a été faite en deux temps : de Thionville à Nancy dès 1970-1972 (surchargée aujourd'hui), le prolongement jusqu'à Dijon ne venant qu'après 1983 ; et la RN 57 bien aménagée de Nancy à Remiremont reste maintes fois incommode de là à Besançon, à Pontarlier et à la Suisse. L'A 35 alsacienne n'a été réalisée que par tronçons successifs entre 1980 et 2002 (et ne compte que 6 ouvrages transfrontaliers de relation à l'HAFRABA badoise). Les couloirs rhénan, mosellan et saônois ont bénéficié d'aménagements fluviaux modernes: Rhin et Grand canal d'Alsace (entre 1950 et 1975) ; Moselle canalisée (entre 1964 et 1979), accueillant de notables trafics et des zones industrialo-portuaires ou logistiques. Toutefois, ce ne sont que des "pénétrantes" pour l'heure non raccordées au système rhodanien. Ces couloirs sont aussi les mieux dotés en grandes lignes ferroviaires et en TER (où sont nés les « Metrolor », « Metrovosges » en 1970 et 1976, « Metralsace » en 1978). L'héritage de «l'étoile de Legrand» a fait prévaloir les améliorations des lignes majeures convergeant sur Paris (il y a encore à faire sur Paris-Bâle), pendant que des attentes subsistent sur les lignes Strasbourg-Lyon ou Dijon, Strasbourg-Metz, Calais-Bâle et sur les liaisons entre la métropole lorraine et Besançon ou Reims, Troyes...

18 La logique des TGV attendus pour 2007 et 2010 (et co-financés par les collectivités territoriales, l'UE, les pays voisins) devrait combiner des dessertes interurbaines plus performantes pour les villes majeures, des irrigations vers les villes moyennes- relais, des accessibilités aux métropoles et « hubs » francilien et lyonnais, et, puisqu'on admet enfin leur intérêt européen, aux réseaux « ICE » et « Rail 2000 » intercités allemands et suisses. Avec les aéroports de l'Est et de ses environs, les horizons s'amplifient - Serace au détriment des lignes déjà signalées comme « médiocres » au sein du Grand Est ? Et si ces infrastructures améliorent les déplacements de voyageurs, les retards pris sur le fret ferroviaire et le «multimodal» renvoient à la question complexe des trafics marchands routiers (où se cumulent les échanges de proximité, interrégionaux, internationaux et de transit).

19 Les accents qu'on vient de mettre sur les chronologies et les tracés visent à surligner des conséquences sur les dynamiques territoriales: ici des ouvertures précoces et multiples, là des désenclavements tardifs ou insuffisants; en phase ou non avec les évolutions économiques générales, ou régionales / locales, et sectorielles... Traits dont la perception est souvent occultée. Et à expliquer le caractère limité, et récent, des systèmes de transport pouvant innerver cette interrégion.

20 La démo-économie de l'après-guerre constitue un autre registre à voir de près. L'époque de "plein emploi ", les progrès de la tertiarisation, l'effacement des frontières (qui, outre la venue d'investissements, facilite les emplois frontaliers), le productivisme 
agricole (migrations vers les villes et les usines)... se combinent alors pour dessiner des contrastes territoriaux remodelés.

21 Aux échelles amples, se constitue un "arc frontalier» (composite), large d'une soixantaine de kilomètres, fait de 18 zones d'emploi denses entre Thionville et Montbéliard: bassins mono-industriels, "portes » fixant la logistique en transports, lieux principalement choisis par les investisseurs extérieurs pour les implantations d'établissements, aires résidentielles de nombre de travailleurs frontaliers, et "chapelet» d'une douzaine des agglomérations moyennes et majeures (plus de 20000 hab.) sur les 28 que compte l'ensemble de nos 3 régions. A l'opposé, Meuse, Haute-Saône et Ouest des Vosges participent de la « diagonale du vide » qui s'accentue sur le sol français dans les années 60-80. Par polarisation urbaine, Nancy et Besançon maintiennent des aires relativement actives, mais que cernent des marges rurales affaiblies. Pour sa part, le Jura comtois s'appuie sur des systèmes productifs locaux sachant s'adapter.

Depuis les années 70, les situations de crise économique générale et sectorielles précoces et prolongées pour le textile, survenant à compter de 1966 dans la sidérurgie, et un peu plus tard dans les Houillères et pour la potasse - la répartition des efforts publics de "reconversion" qui ont suivi, les inflexions des systèmes productifs et organisationnels, les progrès des attractions de main-d'œuvre frontalière, la métropolisation comme processus dominant du développement, concourent à recomposer cette configuration'.

Certains bassins d'emploi, grâce à de nouveaux investissements, sont re-dynamisés (Briey, Sarreguemines), mieux que d'autres (Longwy, Thionville), ou s'agrègent à des aires urbaines (Bassin potassique, Hagondange). Divers territoires restent animés par celles des villes moyennes qui assurent de bonnes intermédiations économiques et sociales (cf. Colmar, Epinal, Saint-Dié, Sarrebourg, Saverne, Bar-le-Duc, Vesoul) ou qui deviennent « relais » de grandes agglomérations (Lunéville, Haguenau)... En plus ample esquisse, métropolisation et développement axial, urbanisation liée aux mobilités accrues concourent à la constitution de deux "régions urbaines" méridiennes. En Alsace ${ }^{10}$, s'enchaînent les zones animées par les métropoles de Karlsruhe, de Strasbourg, de Mulhouse (avec, entre elles, le pays de Colmar) et de Bâle (satellisant Saint-Louis et le Pays des Trois Frontières): fortes densités d'hommes, d'ateliers et de services (nombreuses villes-centres), aires résidentielles frontalières, avec un réseau d'extension sur la Porte d'Alsace par le "réseau de villes Rhin-Sud». S'y atténuent certains dispositifs hiérarchiques, et s'y exercent les attractivités des "synapses" d'accès à la rive droite du Rhin; plusieurs pans du piémont vosgien s'y soudent également. La région urbaine lorraine mosellane est moins continue : demeure une bipolarité majeure (Metz, Nancy), avec des relais (Thionville, Pont-à-Mousson, Lunéville) et des articulations directes au Bassin houiller et Pays de Sarreguemines, d'une part, au sous-ensemble Est-vosgien ayant su se re-dynamiser partiellement (voir Insee-Lorraine, 2003, note 9) d'autre part. A distances, se recomposent des " territoires de marges $»^{11}$ ou intercalaires, des "pays » ruraux, inégalement vivants. Entre ces deux entités, ne ressortent que : "l'Aire urbaine 2000 » de Belfort-Montbéliard et environs, à bases surtout industrielles, solide partenaire du réseau «Rhin-Sud», avec une assiette fonctionnelle qui va de Vesoul au Sud-Alsace (cf. automobile) ${ }^{12}$; et, plus modestement, le Pays de Sarrebourg, relativement autonome. 
déclinaisons des schémas de services, voire expérimentations - cf. Alsace en 1997 pour les transports régionaux de voyageurs, et en 2002 pour la gestion des crédits européens); elles pilotent des réseaux organisateurs de leur territoire (TER, réseaux culturels, technologiques, communication à haut débit par ex.); elles en orientent l'aménagement en "Schéma régional » (cf. Lorraine) ou en "Document stratégique " (cf. Alsace) tous soucieux d'équilibre territorial. Elles s'y efforcent, en propre ou avec le « volet territorial » des contrats de plan et par des dotations allouées à des programmes volontaristes: aux territoires "fragiles" (de montagne, ruraux et industriels en difficultés), aux « villes moyennes » (9 en Alsace) « relais » (16 en Lorraine) ou «pôles régionaux » (6 en Franche-Comté). Ainsi, en Alsace, outre les efforts sur les lycées et les Centres de formation des apprentis liés aux compétences détenues, viennent des aides à la constitution de plates-formes technologiques, à l'intermédiation urbaine par les services (santé, culture, social, administration...) sous forme de contrats. L'enchaînement sur la politique des "pays » (avec Chartes et contractualisations sur projets) ainsi qu'aux «initiatives territoriales », aux diagnostics ou aux programmes territoriaux en est le prolongement. Avec 9 «pays» (dépassant les 40000 hab. hors communauté urbaine de Strasbourg et environs), l'Alsace entière participe à cet étoffement de sous-régions; 14 sont en place en Lorraine et 17 sont constituées en Franche-Comté (souvent modestes et parfois avec des recouvrements). Ainsi, les Régions structurent leur territoire souplement, et sur projets ajustables.

Les Départements, parallèlement, s'efforcent, grâce aux compétences traditionnelles puis transférées, de marquer leurs propres démarches et spécificités. Jusqu'ici, ils ont piloté chacun leur mode de développement local (cf. Lorraine), de gestion des territoires (patrimoine, environnement, tourisme, routes...) - avec toutefois de plus en plus de coordination régionale. Depuis 1992, et surtout 1999, le développement des intercommunalités est en phase avec leur stratégie de gestion « des hommes et des territoires » (nom du programme bas-rhinois lancé en 2003-04). Ainsi les élus locaux promeuvent des «bassins de vie» à travers des EPCI (établissements publics de coopération intercommunale) à fiscalité propre, visant à des économies d'échelle, à répondre aux attentes de proximité (services, lien social, habitat, activités). Ainsi s'amorce un nouveau maillage de "recomposition topologique $»^{13}$. Celui-ci est généralisé en Alsace - 70 EPCI - mais encore faiblement dessiné en Haute-Saône, en vallée du Doubs, sur les plateaux jurassiens, dans l'Ouest des Vosges; cependant, il est inégalement proche des buts énoncés (quand les EPCI ne résultent que d'affinités notabiliaires locales, que de craintes vis-à-vis de la ville dynamique voisine, quand ils tiennent peu compte des éléments fonctionnels de la vie locale - certains sont sans bourg-centre...).

Par ailleurs, sur des logiques «urbaines», se dessinent des «territoires d'agglomération " (ou de communauté urbaine), de gestion et de projet, par adhésion à des compétences - obligatoires et facultatives - et par mutualisation de moyens. L'Est, malgré l'ampleur de ses aires urbanisées, paraît en retard sur ce plan: 
2 Communautés urbaines (Strasbourg, dès 1966, Nancy depuis 1996), et 7 Communautés d'agglomération (Belfort-Montbéliard, Besançon, Metz, " Sarreguemines-confluences », Val de Fensch-Thionville et Mulhouse). Cependant, leurs cadres sont dépassés par les extensions ${ }^{14}$ de la péri-urbanisation résidentielle ou d'activités, pour les transports urbains, en termes de pression foncière... ce qui oblige à des recherches de cohérence avec les intercommunalités (considérées dans les SCOT - schémas de cohérence territoriale) en préparation, par ex.

Enfin, les parcs naturels régionaux méritent mention parmi les territoires délimités, par leurs finalités de sauvegarde des patrimoines associée à une gestion " douce » et « durable » des occupations et activités humaines au sein de leur périmètre : Haut-Jura, Parcs de Lorraine, des Vosges du Nord, des Ballons des Vosges - ces deux derniers en interrégionalité.

La déconcentration des services de l'État, ici comme ailleurs, vient principalement appuyer le rôle des échelons régionaux et départementaux, variablement selon les domaines : soit surtout à celui de la Région, soit avec des services opérationnels plus répartis. Sauf à instaurer des territorialisations spécifiques (massifs montagneux, reconversion industrielle, politique de la Ville, programmes d'aides européennes comme Leader et Interreg...).

Ainsi, des structurations territoriales administrées s'affirment;avec des interdépendances délicates entre niveaux ou "pouvoirs", selon les degrés de consensus politique, de coopération, d'ouverture à la transversalité des approches, de participation active aux choix d'orientation... Et là, les poids du localisme, des autonomies urbaines, des mentalités sont encore forts en France de l'Est ! Il reste que les recompositions établies dans les «territoires institutionnels ", calées soit sur les proximités, sur des structures marquant les exercices de compétences, voire sur des projets, soit sur le souci de plus grande cohérence par résorption de discontinuités ou fragilités régionales comme de meilleures connexités entre réseaux (endogènes et exogènes), doivent aller au-delà de l'organisationnel pour développer des compétences stratégiques, des synergies durables en vue «d'évoluer pour s'adapter » (C. Rolland-May, in note 9). Et passer de ces niveaux à celui de l'interrégionalité «Grand Est » demandera des articulations vigoureuses entre réseaux d'acteurs acceptant la mise en œuvre d'une «gouvernance multi-niveaux » qui se cherche encore; où il sera en outre tenu compte des « territoires géographiques » et des facettes territorialisées des activités économiques, dont les dispositions sont aussi «structurantes» quoique plus floues et elles-mêmes en évolutions.

\section{Quelques interrogations pour l'avenir}

31 Les supports territoriaux et résiliaires conditionnent bien des perspectives de développement en France de l'Est, alors que des fragilités subsistent - cf. supra - et que des inquiétudes nouvelles se font jour.

\section{Questionnements liés à l'économie}

La place dominante déjà signalée des activités et des emplois "de production" n'a guère jusqu'ici été modifiée : ni par les restructurations industrielles lourdes (quasi achevées), ni par les investissements extérieurs réalisés ces dernières décennies. Certes le tissu 
lorrain s'est diversifié ; ceux de l'Alsace et de la Franche-Comté ont été partiellement renouvelés et adaptés (voir note 9); certes de nouveaux secteurs ont pris de l'ampleur : automobile, plasturgie, électronique, équipements industriels, chimie et biotechnologies... Mais, outre que se manifestent désormais des ralentissements dans certains secteurs ou branches, face aux concurrences ou aux aléas de conjoncture, plusieurs délocalisations ou fermetures d'importants établissements inquiètent. Peu bénéficiaires de renforcements en cadres et techniciens - même si des gains substantiels en emplois qualifiés sont à noter - les grandes unités contractent plutôt leurs effectifs; et, en volume d'emploi, les restructurations n'ont pas compensé les emplois perdus. Les PME ou ateliers de taille moyenne, plus souples, se maintiennent mieux mais restent majoritairement « dépendants de stratégies industrielles à grande échelle " (Insee-Lorraine, 2003). Si l'amplification des marchés et la part des firmes étrangères (ou sous leur contrôle) orientent nettement vers l'export, cela inscrit les tissus industriels dans des contextes de forte concurrence.

Les gains technologiques et d'innovation, comme de management, imbriquent désormais davantage métiers industriels et métiers tertiaires (en interne, ou auprès des services aux entreprises); et les "flux tendus" lient les usines à la logistique en transports. Le dynamisme repose de plus en plus aussi sur de bons niveaux de compétences évolutives - dont le "montage " partenarial devient un enjeu vital. Or, l'Est souffre du manque d'envergure de la recherche privée sur place (en 1999, Alsace, 1500 chercheurs, Lorraine, 1 100, Franche-Comté, 1380 : cf. Insee-Alsace, 2003 : Les services... p. 76) ; à côté des nets progrès des services supérieurs de conseil-assistanceétudes (Alsace, 25 000, Lorraine, 20000 ), ceux des services opérationnels (Alsace, 36000 , Lorraine, 37000 ), qui exigent moins de qualification, connaissent un recours accru aux emplois précaires. Avec les postes de travail des transports-logistique (42 000 en Lorraine, 30000 en Alsace), presque tout le tertiaire économique parait développé «dans une logique de prolongement de la production industrielle» (et de ses mutations) et "peu dans les logiques d'investissements immatériels à long terme " (Insee-Lorraine, p. 72). C'est donc tout un « système » qu'il y a lieu de réorienter.

34 L'organisation économique en réseaux, pôles de compétences ou "filières » dans le NordEst amorce ces adaptations: automobile, plasturgie, micromécanique; pour les biotechnologies, un tel réseau est transfrontalier («Biovalley», concernant 400 entreprises dans le Rhin supérieur); des «systèmes productifs locaux» ou des "districts » s'organisent plus localement: on songe à ceux de l'horlogerie, de la lunetterie, du jouet en Franche-Comté par ex. (voir A. Moine, Images de Franche-Comté, $\mathrm{n}^{\circ}$ 28, 2003 et Visage industriel 2001 cité en note 9). Il y a lieu d'y noter les rôles actifs des acteurs publics et de structures incitatives (ici: Apeilor, Alsace-Technologie, Synergie...) ou de transfert : CRITT, Centres de compétences... en tout plus de 50 dans nos 3 régions ${ }^{15}$. Mais on observe que les activités tertiaires marchandes «stratégiques " ont de plus en plus des sièges soit extérieurs, soit insérés dans des structures multirégionales - les métropoles de l'Est s'en trouvent dès lors dépendantes ( $\mathrm{v}$. notes 9 et 10 ) - et que les "technopoles ", par essence fondées sur des réseaux scientifiques et internationaux, de par leurs spécialisations et de par leurs localisations en grandes agglomérations, valorisent davantage celles-ci que les tissus qui leur sont extérieurs sauf en Haute Alsace.

En termes territoriaux, ces éléments ont des répercussions. Des bassins d'emploi sont aujourd'hui « spécialisés » sur la filière automobile : leur horizon en devient dépendant, 
non sans risques. Les aires urbaines de métropoles-technopoles sont plus liées à l'efficacité des réseaux qu'aux ressources de leur territoire. L'appui aux équilibres régionaux qu'apportent les «villes moyennes " se gradue sur leurs capacités à fixer des fonctions d'intermédiation, à assurer des centralités... Bref, des tendances allant dans le sens d'accentuation de certains écarts.

Par la voie des contrats de plan et de ses engagements dans ses domaines " régaliens » (transports et infrastructures, universités et recherche publique...), ses aides directes, son poids dans les décisions et dans l'allocation des aides européennes, les décisions prises en CIADDT, la nécessité de ses contributions aux grands investissements des collectivités territoriales, l'État reste un acteur majeur du développement; toutefois plus pour les échelles d'inscription dans l'Europe ou de programmation en régions qu'à un niveau interrégional.

Polycentrique à sa propre échelle, le Nord-Est participe au processus de métropolisation de manière éclatée. Ceux-ci sont devenus «la substance même»(P. Veltz) des dynamiques économiques. Or, si plusieurs cités s'en nourrissent, aucune n'a l'envergure ni les registres complets d'une "métropole économique ». Strasbourg et Nancy possèdent les services supérieurs publics (sciences, santé, culture...) et des services privés de conseil et d'assistance ou d'études soulignant des rôles d'encadrement, d'organisation relationnelle et d'intermédiation, des structures technopolitaines, mais elles n'ont que peu de poids décisionnel ; Strasbourg y ajoute les sièges d'institutions européennes et la para-diplomatie qui les accompagne. Mulhouse a pour atouts son carrefour bien amélioré, une solide recherche appliquée impliquée dans le transfert technologique. Metz joue de même de son " carrefour-porte » et de son technopole pour se positionner sur "les communications et la communication». Besançon, essentiellement capitale régionale, tente de s'inscrire comme pôle des microtechniques. Mais interviennent aussi des métropoles extérieures outre Paris, bien sûr, et Lyon: Luxembourg, place financière (et fiscale), métropole "offshore» pour les télécommunications et activités télévisuelles (C. Arbaret-Schulz); Sarrebrück en tant que foyer majeur d'une grappe urbaine-industrielle économiquement renouvelée; Karlsruhe, animatrice d'une "région technologique" innovante; Bâle, dont les fonctions dans la banqueassurances, la chimie-pharmacie et la logistique des transports étoffent des flux et réseaux tout à la fois en proximité (Rhin supérieur) et internationaux amples. Il devient dès lors difficile, pour les villes de l'Est, d'atteindre des "masses critiques" génératrices d'entraînements "multicartes". Pour pallier ces faiblesses, quelles stratégies privilégier? Renforcer les spécialisations détenues? Renforcer la qualité des services? S'inscrire dans une dynamique de réseaux sur la base de complémentarités, de partenariats au sein du Grand Est? Ou, en configuration euro-régionale comme le souhaitent Metz (la "Quattropole») ou les villes alsaciennes (Le Rhin supérieur)? Envisager des «métropoles transfrontalières »? Le rapport de la MIIAT Grand Est s'y réfère, mais il resterait à en préciser les systèmes d'acteurs, d'organisation et de gouvernance... Un examen du «concept de développement de l'Agglomération Trinationale de Bâle », élaboré en 2001, est à cet égard significatif ${ }^{16}$. 


\section{Territoires et réseaux transfrontaliers et « eurorégionaux »} Alsaciens vers la Suisse et l'Allemagne, de 60000 Lorrains Palatinat et surtout au Luxembourg (les mouvements de sens inverse sont par contre très limités). Toutefois, les difficultés industrielles de part et d'autre des frontières, malgré la place prise par les emplois tertiaires dans ces flux, les rendent stagnants, affectés aussi par les recours aux contrats précaires et par des questions sociales complexifiées. Les flux de chalandise sont, eux, fonction surtout des centralités urbaines (et de catégories de produits). Gagnent en importance les mouvements liés aux loisirs et à la restauration. Des services communs d'information et de consommation, des actions structurées autour de la formation et de l'emploi, et des "clubs d'entrepreneurs" renforcent ces osmoses, tandis que naissent des «zones d'activités transfrontalières »: Belval (tertiaire), « Eurozone » proche de Sarreguemines (1995, gérée depuis 2000 par un groupement local de coopération offrant le choix de fiscalité et de droit français ou allemand), projets à Lauterbourg, en Sud Alsace. Enfin, relations économiques étroites et degrés élevés d'urbanisation en Sarre, Bade et région bâloise stimulent des acquisitions foncières et immobilières étrangères sur le sol français : en 1999, on dénombrait 15800 Allemands et 3300 Suisses résidant en Alsace ; les Sarrois installés en Moselle-Est étaient en 1997 quelque 17000 selon M. Ramm (Mosella, 1999, 3-4, p 129).

41 Cette dynamique suscite des développements des systèmes de transports: tramway de Sarrebrück à Sarreguemines (1997); "S. Bahn» bâlois ou "Ligne verte» entre Mulhouse, Bâle et Frick (1997); réactivation de voies ferrées entre Lauterbourg et Wörth-am-Rhein, entre Wissembourg et Winden, entre Mulhouse ou Colmar et Fribourg-en-Brisgau (en cours ou en projet) ; « MetroRhin » cadencé Strasbourg-Kehl et Offenbourg (2001-02). On en vient également à l'organisation spatiale coordonnée d'aires transfrontalières. Dans le Pôle européen de développement de Longwy (PED), d'importants efforts publics ont, par delà les restructurations industrielles de la crise sidérurgique puis de délocalisations brutales, porté sur le logement, le traitement des friches, et encouragé la constitution d'une Association transfrontalière (1996) dotée d'une Charte d'agglomération et d'un $\mathrm{SIG}^{17}$. Une Association "Sarre- Moselle Avenir " entre le "Stadtverband Saarbrücken » et le District de Sarreguemines coordonne les problèmes d'environnement, de gestion spatiale et les liens culturels. Des Plans locaux 
d'habitat transfrontaliers naissent en Alsace du Nord sur la Bande rhénane; le Pays de Saint-Louis s'inscrit dans la dynamique de l'ATB (cf. note 16). Et, officiellement, s'élabore un ambitieux "Eurodistrict» Strasbourg-Ortenau (2003-04). Dans toutes ces organisations, place est faite à des actions soulignant des partages d'identité, des liens socio-culturels, et invitant aux projets communs. Ces derniers visent les services, la santé, la gestion des espaces et des risques...mais des distinctions seraient à faire selon les «écarts de primauté » entre facteurs de dynamisme, entre acteurs incitatifs, entre collectivités partenaires de poids inégal...

Les stratégies "eurorégionales» de l'Est sont, elles, à la fois territoriales et résiliaires. Elles proposent un deuxième niveau de coopération "d'échelle régionale ", où se mêlent des périmètres institutionnalisés et des dispositifs plus variables, des aspects d'organisation de vie sous-régionale et des réseaux de plus grande envergure ${ }^{18}$.

Ainsi, la Communauté de Travail du Jura (CTJ), née en 1985 sur initiative suisse, associe diversement la Région de Franche-Comté et les Cantons du Jura, de Berne, de Neuchâtel et de Vaud. Même lorsqu'elle se dote en 1993 d'une Charte et d'un programme opérationnel doté de soutiens de l'État, des fonds européens puis confédéraux, l'essentiel concerne des rapports économiques de proximité (industrie horlogère, tourisme, formation...) et de circulation, pour 3 sous-ensembles: Haut Jura, pays de Pontarlier et de Morteau-Maîche/Le Locle et la Chaux de Fond, pays de Delle et canton du Jura. L'aspect résiliaire est d'abord celui des systèmes productifs locaux. Néanmoins aujourd'hui, il s'étend aux échanges entre universités ou laboratoires, aux dimensions culturelles et d'interconnaissance, amorçant peut-être des bases d'un réseau des grandes villes par delà une relance des armatures locales. Pourtant, l'avenir y dépend amplement de la capacité suisse à garder son avance technologique horlogère et micromécanique, à "tenir ses frontières " ainsi que les équilibres de son système urbain majeur.

L'ensemble "Rhin Supérieur ", plus étoffé et plus ample, superpose une organisation en 3 " entités-territoires » et des compositions de réseaux. Au Sud, la "regio TriRhéna », conçue initialement par des intérêts privés bâlois (1963), s'est constituée et régulée dans le cadre de la Conférence du Rhin supérieur et des programmes Interreg, par association du Haut-Rhin, du Bade du Sud et de la Suisse du NW. A l'aménagement coordonné du territoire et aux rapports économiques et socio-culturels locaux, il ajoute des éléments résiliaires: des connexions de systèmes de transports, l'accès à l'EuroAirPort commun, les entreprises participantes de Biovalley déjà citée, la mise en réseau d'organes de transfert ou de formation, comme des musées, des foires (Bâle, Mulhouse, Colmar, Fribourg). Sa dynamique sur l'économique est patente. (voir article de B. Reitel dans ce $n^{\circ}$ ). Au Nord, «l'espace Pamina» (Palatinat du Sud, Mittlerer Oberrhein - région de Karlsruhe - Nord de l'Alsace), lancé en 1988 sur les projets limités aux proximités d'une aire située entre Strasbourg et Karlsruhe, devient maintenant plus ample et plus ambitieux, d'autant que les deux grandes agglomérations voisines s'impliquent davantage à la vision d'aménagement plus complète que veut promouvoir son syndicat mixte créé en 2003. Là, les objectifs se fixent sur les maillages territoriaux et les services. Entre les deux, une région "Centre», dont "l'absence de solidarité spontanée» (dit son Président) a gêné jusqu'ici le développement de projets, va prendre plus d'ampleur avec le Schéma de cohérence territoriale de Strasbourg-Ortenau. 
45 Par delà, l'échelle du Rhin supérieur dans sa globalité se construit aussi par des réseaux: ceux d'un schéma commun des transports et de logistique, après la constitution d'un réseau des universités (EUCOR, 1984-89) et d'Ecoles supérieures bilingues, de recherches, d'un réseau des foires, des congrès (EurocongrèsRhin), de promotion des musées - musées sans frontières... Donc un ensemble alliant maillages et complémentarités, objectifs d'aménagement et de développement coordonnés, souci d'entretenir «un jardin de villes». Mais aussi l'ambition de promouvoir «une métropole trinationale décentralisée » (Livre Blanc, 2001, p. 27), face à des inquiétudes sur l'avenir d'une région qui craint d'être à l'écart des grands axes et pôles européens. «Saar-Lor-Lux» est enfin une «eurorégion» précocement esquissée. Dans sa configuration officielle interétatique, à l'ensemble Sarre-Lorraine-Grand Duché de Luxembourg, se sont ajoutées la Rhénanie-Palatinat et la Province belge de Luxembourg (Wallonie orientale). C'est la «Grande région». Dans une majorité de ses réalisations, le «noyau majeur » implique surtout la Sarre, le Luxembourg et le Nord de la Lorraine : là sont ses points d'origine : l'ancien "triangle d'or de la sidérurgie " (C. Arbaret-Schulz), relancé par la CECA en 1958, et ses bases économiques renouvelées (consortium bancaire trinational, World Trade Center Metz-Sarrebrück, organes interconsulaires et de transfert technologique, bourses de sous-traitance..., là ont été nouées les premières coopérations interuniversitaires (diplômes communs instaurés dès 1978, dans le cadre d'une Charte d'" université sans murs" associant Metz et Sarrebrück. Actuellement, le réseau concerne aussi les universités et écoles nancéiennes et celle qui naît à Luxembourg. Instaurée en 2000, une « Quattropole » met en réseau les activités de télécommunications, de multimédias, d'informatique haut débit de Metz, Sarrebrück, Luxembourg et Trèves. Un tel réseau de grandes villes concurrence-t-il celui que voudrait promouvoir Nancy entre villes du Sillon mosellan ? En tout cas, Saar-Lor-Lux relève d'une logique d'alliances surtout urbaines, multilatérales, plus que d'une logique de réticulation telle qu'incluse dans le cadre du Rhin supérieur.

Dans les trois cas, les eurorégions signalent des stratégies (exprimées par des réseaux et par des perceptions "d'avenir commun») d'espaces-frontières réagissant à des positions de «marges» (ou craignant de s'y trouver) au regard des grandes trames et polycentrismes majeurs du Nord-Ouest européen. Si assez aisée paraît la structuration locale des rapports transfrontaliers, plus difficilement se fixe une prééminence des réseaux eurorégionaux: les relations économiques des firmes, scientifiques des laboratoires et universités, les grands systèmes de transport et de logistique « européens »... dépassent ces cadrages. Certes le Grand Est peut y trouver des «alliés partenaires ", des éléments de signalétique, d'ancrage et d'image renouvelés à l'égard de l'Hexagone et de l'espace rhénan, voire européen. Encore y a-t-il à renforcer des articulations et des complémentarités en interne et avec ses espaces voisins pour mieux aborder les mutations d'aujourd'hui et de demain. 


\section{NOTES}

1. CIAT-DATAR (1990). Orientations et enjeux de la politique nationale d'aménagement du territoire. Doc. Fr., 11 p. Explicitations dans : Leclerc R. et al. : (1996). Les régions au futur. Ed. de l'Aube, 142 p. et $\mathrm{N}^{\circ}$ spécial de la revue Hommes et Terres du Nord, 1996 « Prospective pour 2015, de l'Europe aux régions $"$.

2. On sait que, émergentes en 1955-56, ébauchées par la régionalisation partielle du Plan (1965-70), elles ont d'abord été validées comme Etablissements publics régionaux (EPR) en 1972 ; leur statut de collectivité territoriale n'a été obtenu qu'en 1983 (fonctionnement de fait en 1986) avec les Lois Deferre (décentralisation) et Rocard (contractualisations du Plan entre État et Régions).

3. «Eurorégions": voir Demazière C. et al., (1996). Du local au global. L'Harmattan, 382 p. $\mathrm{n}^{\circ}$ spécial de Hommes et Terres du Nord, 3, 1998, «Eurorégion »; Nonn H. (2001). L'aménagement du territoire en Europe occidentale, éd. Ellipses, 160 p. L'interrégionalité « hors proximité » est illustrée par la démarche rhône- alpine au sein du «Quadrige européen » lancé en 1988 - voir Kukawa $\mathrm{P}$ (1999). Le Quadrige européen. Presses Universitaires de Grenoble.

4. DATAR (2002). Collection «Aménager la France en 2020 » publiée par la Documentation Française : Contributions de l'État à de nouveaux enjeux territoriaux : Le Nord, 123 p. Le Grand Bassin Parisien, 311 p. Le Grand Sud Est, 169 p. Le Grand Est, 160 p. Mangin C (2002). Les coopérations interrégionales. Doc. Fr. collection « Territoires en mouvement », 108 p.

5. Nonn H. (1996). «Le Grand Est dans la démarche d'aménagement du territoire » in : Hommes et Terres du Nord n³, p. 158-165 (résumant des rapports à la Datar).

6. Indications dans: Schwab R. (1980). De la cellule rurale à la région: L'Alsace 1825-1960, éd. Ophrys ; Lassus F. (1997). "Canaux et chemins de fer aux XIX ${ }^{\mathrm{e}}-\mathrm{XX}$ e S. », Images de Franche-Comté, $\mathrm{n}^{\circ}$ 16, p. 1-4 ; Gérard C. (1977). in Collectif : Histoire de la Lorraine t. 8, éd. Mars et Mercure, ColmarWettolsheim, p. 86 et suiv. ; Caro et al. (2002). La politique d'aménagement du territoire (1-2 ${ }^{\circ}$ parties, par F. Caron, C. Bouneau, P. Berion et J.F. Langumier ; Presses Universitaires de Rennes.

7. Baudin F. (1992 et 1993). Histoire économique et sociale de la Lorraine 1. Les racines, 2. L'essor ; Presses Universitaires de Nancy et éd. Serpenoise ; Houles-Cornet A (1986). Un espace industriel en voie de restructuration : le bassin potassique d'Alsace. Thèse $3^{\mathrm{e}}$ Cycle, Géographie, Strasbourg 1, $330 \mathrm{p}$. et Meyer P. (1963). «Aspects géographiques d'une main-d'œuvre industrielle : les mineurs des MDPA », Revue Géographique. de l'Est, n 4, p. 343-369; Dezert B. (1969). La croissance industrielle et urbaine de la Porte d'Alsace. éd. SEDES, $520 \mathrm{p}$.

8. Collectif (1980). La France des villes: Nord et Nord-Est, Vol. 3 par H. Nonn et P. Claval. Doc. Fr ; Dion R.M. (1983). La région urbaine de Nancy. multigr. 934 p. ; Bonnefont J.C. (1973). Nancy et son agglomération. Doc. Fr.; Reitel F. (1983). in Géographie de la Lorraine. Chap. concernant Metz (p. 11-28 et 413-432) éd. Serpenoise.

9. INSEE-Lorraine (2003). La Lorraine face à son avenir (J. Creusat, dir.), 124 p.; INSEE-Alsace (2000). L'Alsace industrielle. - renouvelant une étude de 1991 parue sous le même titre, 74 p.; INSEE- Alsace (2003). Les services marchands en Alsace; 89 p. ; INSEE Franche-Comté (+ DRIRE et Région) (2001). Visage industriel de la Franche-Comté, 74 p. et Nardy J.P. (1995). "Evolution de l'industrie comtoise » in Images de la Franche-Comté, $\mathrm{n}^{\circ} 18$ et 20 ; Nonn H. et Heraud J.A. (1995). Les économies industrielles en France de l'Est, tissus et réseaux en évolutions... Pr. Univ. de Strasbourg, 305 p. ; Dezert B. (v. note 7) ; Grosdidier F. (1996). La reconversion, une ardente obligation. Rapport officiel, Doc. Fr., 230 p.

10. Nonn H. (1999). Villes et aménagement régional en Alsace. Doc. Fr. 246 p., bibl.

11. Groupe RITMA-M.S.H. Strasbourg (2001). Regards croisés sur les territoires de marge(s). Presses Universitaires de Strasbourg, 239 p.; Rolland-May C. (1993). Les zones rurales fragiles en Lorraine. 
Rapport à la Préfecture de Lorraine, 27 p. multigr ; n de la Revue Géographique de l'Est 1996, 3-4, « Analyse spatiale et développement local en Lorraine » (C. Rolland-May, S. de Ruffray...) ; $\mathrm{n}^{\circ}$ de la revue Mosella, 1999, 3-4 « La Lorraine et ses bordures » (mêmes auteurs).

12. Woessner R. (2000). Mythe et réalité de l'espace «Rhin-Rhône »: la dynamique industrielle comme facteur de recomposition territoriale. Presses Universitaires de Franche-Comté, 360 p. + nombreux articles dans la revue Entre Rhône et Rhin. CRCI, CCI, DRIRE, ADIT (2001). La filière automobile en Alsace et en Franche-Comté. 2 Cahiers, 92 et 193 p.

13. Rolland-May C. (2000). "Géostratégie de la recomposition des territoires : cas particulier... L'espace «médio-lorrain» autour de Pont-à-Mousson" Revue Géographique de l'Est. n 4, p. 155-173, et Id. (2000) : L'évaluation des territoires, concepts, modèle, méthode. Hermès-Sciences, $400 \mathrm{p}$. 14. Hulbert F. (2001). «La recomposition politique des espaces urbains en France et le cas de l'agglomération de Metz ", in : Mosella, $\mathrm{n}^{\circ}$ 3-4, p. 375-391. AURM (Agence d'urbanisme de la région mulhousienne) (2002) : Périmètre d'étude pour le Pays de la région mulhousienne, 23 p. multigr. Reitel B. (1999) «Stratégie urbaine et dimension territoriale : le cas des trois principaux pôles urbains d'Alsace ", Regio Basiliensis, p. 17-42.

15. Nonn H. (1995). Prospective Grand Est : Dimensions interrégionales et internationales dans les Schémas régionaux... Enseignement supérieur, Recherche et Transferts de technologie. 58 p. + annexes, multigr.

16. ATB (2001). Concept de développement et projets-clés pour l'Agglomération Trinationale de Bâle. 177 p. Reitel B. (2002). « Asymétrie des formes et différentiels morpho- logiques dans l'ATB : des propriétés de ville- frontière?", in Reitel et al. (2002). Villes et frontières; Anthropos-Villes, p. 84-96 + bibliogr.

17. Schulz C. (1996). «L'agglomération transfrontalière du Pôle Européen de Développement Longwy-Rodange-Athus", Revue Géo- graphique de l'Est, n² 2, p. 133-150; Auburtin E. (1999). «L'agglomération transfrontalière de Longwy, un exemple de maîtrise territoriale du phénomène frontalier? » Mosella, $\mathrm{n}^{\circ}$ 1-2, p. 161-173; Ait-Oumeziane A. (2000). « Reconversion économique et construction d'un territoire transfrontalier", Annales de Géographie, $\mathrm{n}^{\circ} 611$, p. 65-83.

18. Moine A. et Genre-Grandpierre C. (1999). "L'Arc jurassien franco-suisse, une communauté de destin ", in Images de Franche-Comté, $\mathrm{n}^{\circ}$ 20, p. 14-17, et Moine A. (2002). « Les villes frontalières de l'arc jurassien franco- suisse » in Villes et frontières (op. cité, note 16), p. 69-83. ECOHRS (1998) Étude d'un cadre d'orientation pour le Rhin supérieur. ADEUS (1999). Lire et construire l'espace du Rhin supérieur. Ed. La Nuée Bleue, Strasbourg. Actes du $7^{\mathrm{e}}$ congrès tripartite "Aménagement du territoire du Rhin supérieur, Neustadt. Nov. 1999, 3 fascicules de restitution des groupes de travail. Reitel B. (1997). Esquisse d'une armature urbaine pour l'Alsace et le Rhin supérieur. Rapport au Conseil Régional d'Alsace, 45 p. Conférence franco-germano-suisse (2001) Pour un avenir commun de l'espace du Rhin supérieur, Livre Blanc, 46 p. Gaunard M.F. (1998). «Réflexions sur une stratégie d'organisation territoriale des espaces urbains frontaliers : agglomérations ou réseaux de villes transfrontalières dans l'espace Saar-Lor-Lux ", Hommes et Terres du Nord, n ${ }^{\circ} 1$, p. 17-24 ; ArbaretSchulz C (1993). «Sarre-Lorraine- Luxembourg: vers un réseau de villes transfrontalier? » in : Sallezet al., Les villes, lieux d'Europe. Datar-éd. De l'Aube, p.139-146 ; Id. (1999). «Histoires de frontières et de villes-frontières" Mosella $\mathrm{n}^{\circ}$ 1-2, p. 125-132. PRELUDES, Les Cahiers (1997). «L'espace économique transfrontalier Lorraine-Sarre-Luxembourg et Belgique » et «Villes et coopération transfrontalière »; Schulz C. (1999). "Saar- Lor-Lux, un espace modèle pour l'intégration européenne? », Bull. A.G.F., p. 397- 407. 


\section{RÉSUMÉS}

Le concept récent de "Grand Est » suscite une réflexion sur l'organisation des territoires et de réseaux dans la France de l'Est, allant de l'histoire contemporaine aux situations actuelles et aux perspectives de demain. Elle met en regard les places majeures que tiennent les écarts internes ou locaux de développement, le souci des équilibres régionaux, les rapprochements transfrontaliers et les enjeux liés aux horizons plus étendus. L'interrégionalité "Grand Est ", comme les "eurorégions" auxquelles adhèrent ses composantes, répondent à des préoccupations d'insertion dans des espaces plus amples; mais, dans les deux cas, avec des partages d'enjeux et de capacités résiliaires et d'entraînement incomplets, qui doivent compter avec les recompositions territoriales géographiques, économiques et institutionnelles en cours.

The recent concept of "Great East" raises questions about the organisation of territories and networks in eastern France, within the context of contemporary history, present circumstances and future prospects. It contrasts the major places with internal or local disparities in development, concern about regional balance, cross-border reconciliation, and the possibilities associated with even wider horizons. The interregionality of "Great East", like the "euroregions" to which these components adhere, addresses the preoccupation with integration into more ample spaces; but, in both cases, with incomplete sharing of the possibilities, of networking capacities and of training, that must take account of current geographical, economical and institutional territorial adjustments.

Das heutige Konzept des "Grossen Osten" führt zu einer Überlegung über die Organisation der Territorien und Netze in Ostfrankreich, die von der Zeitgeschichte über die gegenwärtige Lage bis $\mathrm{zu}$ den Perspektiven von morgen führt. Es zeigt die internen oder lokalen Entwicklungsunterschiede auf, die Sorge um regionalen Ausgleich, die grenzüberschreitenden Annäherungen und die mit den erweiterten Horizonten verbundenen Probleme. Die Interregionalität des "Grossen Osten" wie die "Euroregionen", denen seine Glieder angehören, ergeben sich aus der Sorge vor der Einbeziehung in grössere Räume - in beiden Fällen aber verbunden mit der Teilung der Arbeitsplätze und Wohnkapazitäten und mit unvollständiger Ausbildung. Dabei muss mit den gegenwärtigen territorialen geographischen, ökonomischen und institutionellen Umbildungen gerechnet werden.

\section{INDEX}

Mots-clés : Grand Est, interrégionalité, réseau, territoire

Schlüsselwörter : Grosser Osten, Interregionalität, Netz, Territorium

Keywords : Great East, interregionality, network, territory

\section{AUTEUR}

\section{HENRI NONN}

Professeur honoraire (géographie et aménagement) de l'Université Louis Pasteur de Strasbourg, 14 rue du Canal, 67400 Illkirch 